Research Paper

\title{
Worse Prognosis in Papillary, Compared to Tubular, Early Gastric Carcinoma
}

\author{
Huiping $\mathrm{Yu}^{1 *}$, Cheng Fang $1,2,4^{*}$, Lin Chen¹, Jiong Shi1, Xianshan Fan1, Xiaoping Zou², Qin Huang1,2,3凶 \\ 1. Department of Pathology, Nanjing Drum Tower Hospital, 321 Zhongshan Road, Nanjing, P.R.China. \\ 2. Department of Gastroenterology, Nanjing Drum Tower Hospital, 321 Zhongshan Road, Nanjing, P.R.China. \\ 3. Department of Pathology and Laboratory Medicine, Veterans Affairs Boston Healthcare System and Harvard Medical School, 1400 VFW Parkway, West \\ Roxbury, MA 02132, U.S.A. \\ 4. The current affiliation: Department of Gastroenterology, Zhejiang University affiliated Second Hospital, Hangzhou, China. \\ * These Authors are co-first authors. \\ $\triangle$ Corresponding author: Qin Huang, MD, PhD, FCAP. Department of Pathology and Laboratory Medicine, Veterans Affairs Boston Healthcare System and \\ Harvard Medical School, 1400 VFW Parkway, West Roxbury, MA 02132, U.S.A. Email: qinhuang0122@gmail.com. \\ (C) Ivyspring International Publisher. This is an open access article distributed under the terms of the Creative Commons Attribution (CC BY-NC) license \\ (https://creativecommons.org/licenses/by-nc/4.0/). See http://ivyspring.com/terms for full terms and conditions.
}

Received: 2016.08.23; Accepted: 2016.10.29; Published: 2017.01.01

\begin{abstract}
Purpose: Papillary early gastric carcinoma (EGC) is uncommon but shows worse prognosis in our most recent study in a Chinese population with unknown reasons. The aim of the present study was to further investigate risk factors for worse prognosis in patients with papillary adenocarcinoma, compared to those with tubular adenocarcinoma.

Methods: We searched the electronic pathology databank for radical gastrectomy cases over an 8-year period at a single medical center in Nanjing, China, and identified consecutive 240 EGC cases that were classified as either papillary $(n=59)$ or tubular $(n=181)$ EGC tumors in accordance with the World Health Organization (WHO) gastric cancer diagnosis criteria. We investigated and compared clinicopathologic risk factors for prognosis between papillary and tubular EGC groups. All patients were followed up and their 5-year survival rate was compared statistically with the Kaplan-Meier method with a log rank test.

Results: Compared to tubular EGCs, papillary EGCs were significantly more common in elderly patients, more frequently occurred in the proximal stomach with protruding/elevated growth patterns, submucosal invasion, and a micropapillary component. Although lymphovascular invasion (16.9\%), nodal (13.6\%) and distant (11.8\%) metastases in papillary EGCs were more frequent than those $(8.3 \%, 7.2 \%$, and $3.7 \%$, respectively) in tubular EGCs, the differences approached but did not reach statistically significant levels. Significant risk factors for nodal metastasis included lymphovascular invasion in both EGC groups, but the ulcerative pattern and submucosal invasion only in tubular EGCs. The 5-year survival rate was significantly worse in papillary (80.5\%) than in tubular (96.8\%) EGCs.

Conclusions: Compared to tubular EGCs, papillary EGCs diagnosed with the WHO criteria in Chinese patients were more frequent in elderly patients, proximal stomach and showed the significantly worse 5 -year survival rate with more protruding/elevated growth patterns and the micropapillary component. Further studies in larger samples are urgently needed to validate these findings for precision individualized EGC patient management.
\end{abstract}

Key words: stomach, early gastric carcinoma, papillary, prognosis, nodal metastasis.

\section{Introduction}

The World Health Organization (WHO) classifies gastric adenocarcinoma into 5 main types: tubular, papillary, mucinous, poorly cohesive, and mixed, in which the most common type is tubular, whereas papillary adenocarcinoma is uncommon [1]. Papillary adenocarcinoma is characterized by finger-like projections lined with neoplastic columnar cells surrounding a fibrovascular core, and associated 
with small micropapillary and tubular components in some tumors [1]. Both papillary and tubular adenocarcinomas are also grouped as "intestinal" (versus "diffuse", according to the Lauren classification) [2] or "differentiated" (versus "undifferentiated", based on the Japanese classification) [3] gastric carcinoma.

Gastric carcinoma remains one of the most common cancers and the leading cause of cancer deaths worldwide [4], especially in China with over $43 \%$ of worldwide gastric cancer cases [5]. At present, the only hope for improving patient survival is the early detection and resection of early gastric carcinoma (EGC), which is defined as invasive carcinoma in the depth up to the submucosal layer, regardless of the nodal status [1]. Both papillary and tubular adenocarcinomas at the early pT1 stage in the size of smaller than $2 \mathrm{~cm}$ without ulceration or lymphovascular invasion are considered to have a minimal or no risk for nodal metastasis on the basis of study results published in 2000 by Japanese investigators [6]; as such, EGC tumors are suitable for endoscopic resections, such as endoscopic submucosal dissection (ESD), because of minimal injury and safe to EGC patients, as recommended by both Japanese and Chinese clinical practice guidelines $[7,8]$.

In our most recent study on EGC in Chinese patients [9], we found surprisingly that the patients with papillary EGC had worse prognosis than those with tubular EGC; in addition, a high nodal metastatic rate was found in half cases of papillary EGCs with a small micropapillary component [9]. In fact, Japanese and Korean investigators have reported previously a considerable proportion of papillary adenocarcinoma cases with liver and nodal metastases and worse prognosis [10-13]. While those investigators follow the Japanese EGC diagnostic criteria in which invasion is not considered the most important criterion for carcinoma diagnosis [14], the prognosis and risk of nodal metastasis in papillary EGC remains unclear in Chinese patients, in whom EGC is diagnosed primarily with the WHO criteria. The purpose of this study was to further analyze our EGC database previously established at a single high-volume medical center in China to delineate and compare the prognosis and risk factors for nodal metastasis between papillary and tubular EGCs, which were diagnosed with the stringent WHO criteria [1].

\section{Methods and Material}

\section{Patient Groups}

In the prospectively built pathology electronic databank with gastric cancer resection cases over an 8-year period from 2005 to 2012, we found and retrospectively reviewed a total of 3176 pathology reports on radical gastrectomy and identified consecutive 379 EGC cases with lymphadenectomy. Each EGC pathology report along with all histology slides was retrieved and investigated. Histology types of EGC were confirmed or modified strictly with the WHO diagnostic criteria [1]. Excluded were the cases with synchronous or gastric stump tumors and the cases without slides for review or tissue blocks for recuts. The cases with the diagnosis of papillary or tubular adenocarcinoma were selected for the comparison study and divided into the two groups, respectively. The patient demographic information was extracted from the pathology report. Each endoscopic report along with all endoscopic and gross images of selected EGC tumors was studied for the information of tumor location and endoscopic tumor growth patterns, which were categorized in accordance with the Paris classification [15]. Patient consent to surgical resection and clinical research of the tumor was collected with the signature prior to the surgical resection. The study protocol was approved by the Medical Ethics Committee of the Nanjing Drum Tower Hospital in China.

\section{Pathology Investigation}

With a standard pathology tissue processing protocol [9], all surgical resection specimens were carefully evaluated for tumor location, size, shape, color, surface appearance, invasion depth, and consistency. The stomach was divided into proximal and distal regions, as previously described [16]. The proximal stomach, also known as gastric cardia, was defined as a narrow region of about $3 \mathrm{~cm}$ below the gastroesophageal junction grossly[16,17], and confirmed microscopically by histologic landmarks, such as the distal ends of the squamous epithelium, multi-layered epithelium, and deep esophageal glands and ducts[16]. All other parts of the stomach, such as corpus, antrum, and pylorus, were grouped as the distal stomach. The tumor maximal size was measured manually in the formalin-fixed specimen and recorded. Microscopic examination was carried out by at least two senior pathologists with special interest and experience in gastrointestinal pathology. The endoscopic ulcerative pattern was re-assessed by histologic identification of disruption of the lamina propria to the muscularis mucosae and beyond. Guided by the WHO diagnostic criteria [1], we classified papillary adenocarcinoma into two major morphologic growth patterns: 1) the conventional villiform pattern on well oriented sections (Figure 1), or as free-floating villi with a fibrovascular core or lamina propria, if occupied more than $50 \%$ of the 
estimated tumor volume on the same slide[10,11];2) the micropapillary pattern, featuring small pseudopapillary tumor clusters surrounded by empty lacuna spaces without a fibrovascular core (Figure 2), if found in over $5 \%$ of the estimated tumor volume[9]. In contrast, tubular adenocarcinoma demonstrated a more versatile growth spectrum, including tubular, acinar, cribriforming, anastomosing, dys-union, microcyst, spiky branching, and budding patterns, etc. In each EGC tumor, the invasion depth was semi-quantitatively determined on the best oriented tumor section and divided among 4 subgroups: lamina propria (M2), muscularis mucosae (M3), and superficial $(<500 \mu \mathrm{m})(\mathrm{SM} 1)$ or deep $(>500 \mu \mathrm{m})(\mathrm{SM} 2)$ submucosal layer. In tumors with submucosal invasion, lymphovascular and perineural invasion was also assessed. In cases with equivocal lymphovascular invasion on hematoxylin-eosin stained sections, routine immunostains for D2-40 and CD34 were carried out with both negative and positive controls in each run, as previously described [9]. Lymph node metastasis was evaluated with manual counting lymph nodes retrieved and the nodes with metastasis, identified on a routine hematoxylin-erosin stained tumor section in each case. The information on tumor distant metastasis was acquired by reviewing the patient electronic medical record and most recent follow-up clinical notes [9].

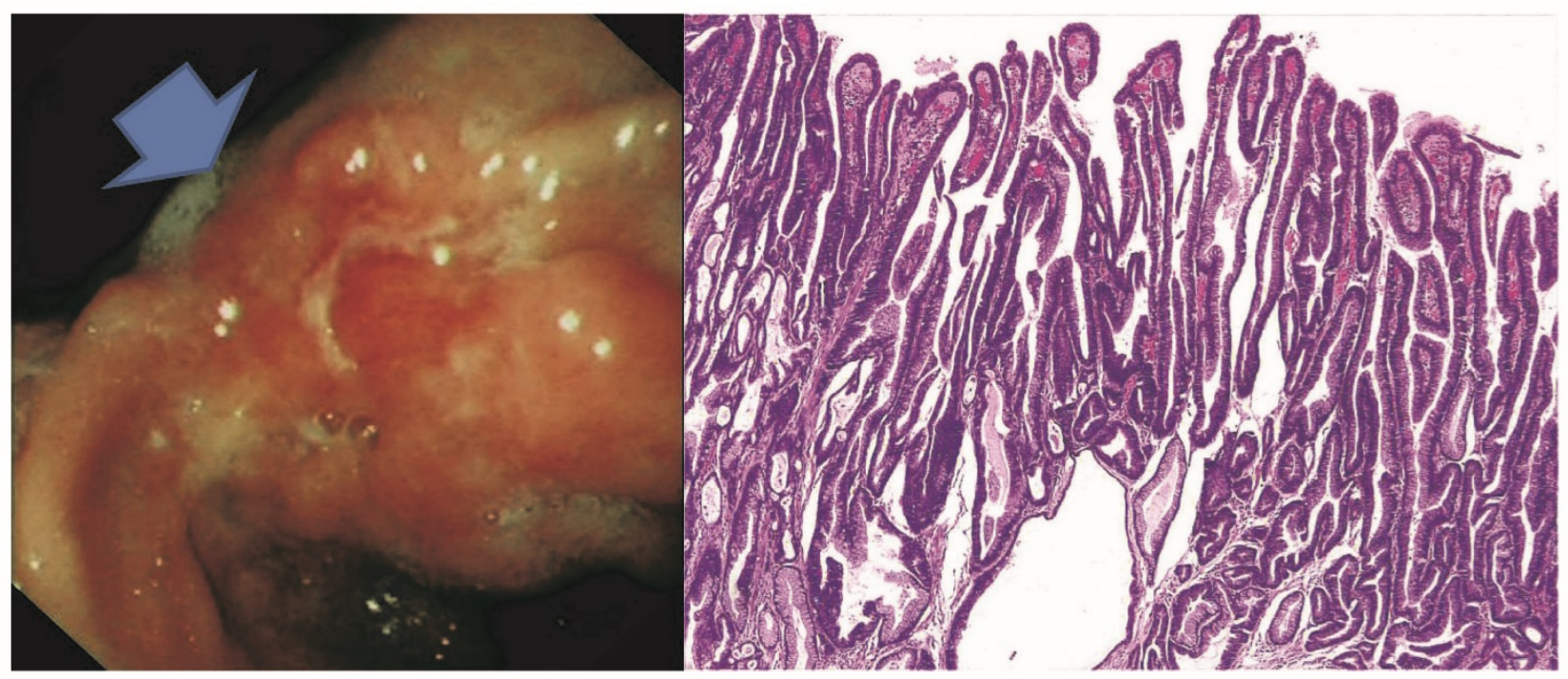

Figure 1. Representative papillary early gastric adenocarcinoma in the gastric angularis incisura with the endoscopic O-IIC growth pattern in A. By histology in B, the tumor shows a villiform growth feature with finger-like papillary projections and fibrovascular cores.

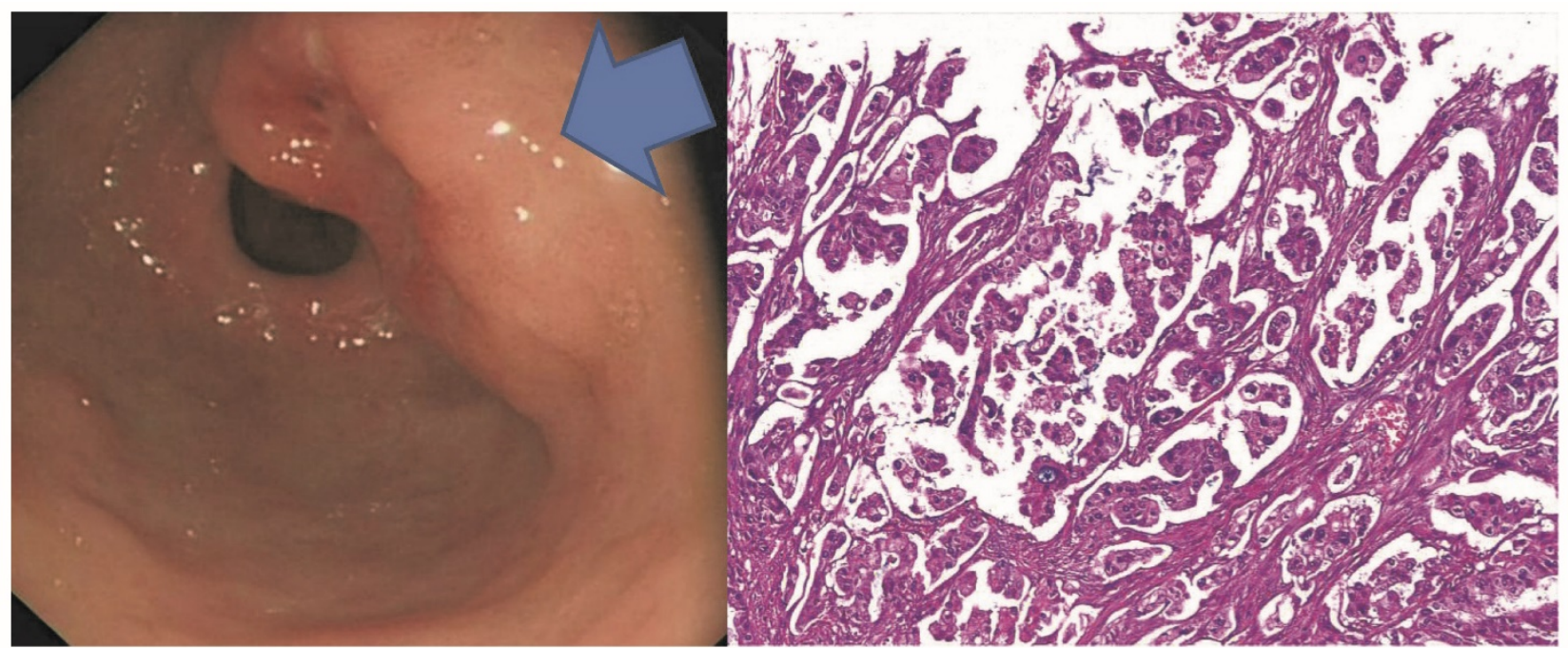

Figure 2. Representative micropapillary early gastric adenocarcinoma in the gastric antrum with the endoscopic O-IIA/O-IIC grow patterns in A. Microscopically, the tumor shows micropapillary features without fibrovascular cores but demonstrates empty spaces surrounding each tumor micropapilae. 


\section{Statistical Analysis}

Differences in clinicopathology, endoscopy, and survival between papillary and tubular EGC groups were assessed with the Chi-square and Student's $t$ tests. Patient survival was calculated from the month of EGC diagnosis to the month of the last follow-up if alive, or death of all causes. Survival rates were estimated with the Kaplan-Meier method with a log rank test. Univariate and multivariate analyses were carried out with the logistic proportional hazards model to determine independent risk factors of nodal metastasis. The Statistical Package for the Social Sciences (SPSS, version 19.0, Chicago, IL, the United States of America) was employed for all statistical analyses. $P$ values smaller than 0.05 were considered as statistically significant.

\section{Results}

Among 379 EGC cases, 240 were qualified for the study and divided into the papillary $(\mathrm{n}=59,24.6 \%)$ and tubular $(\mathrm{n}=181,75.4 \%)$ groups.

\section{Comparison in Clinical, Endoscopic, and Pathologic Characteristics}

As shown in Table 1, there were no statistically significant differences in gender and tumor size between the two EGC groups. However, patients in the papillary group were significantly older for the age over 61-year category $(78 \%)$, compared to those in the tubular $(58.6 \%)$ group $(p=0.007)$. Similarly, the papillary group demonstrated significantly more frequent proximal gastric location $(55.9 \%$ vs. $28.7 \%)(p$ $<0.001$ ), protruding/elevated (OI + OIla) endoscopic growth patterns $(66.1 \%$ versus $39.2 \%)(p<0.001)$, submucosal invasion $(64.4 \%$ versus $43.6 \%)(p<0.05)$, and the micropapillary growth pattern $(6.8 \%$ versus $0)(p<0.003)$. Lymphovascular invasion was more than twice as common as in the papillary $(16.9 \%)$ than in the tubular (8.3\%) group, and the difference almost reached a statistically significant level $(p=0.059)$. Likewise, although nodal metastasis was also more frequently discovered in the papillary $(13.6 \%)$ than in the tubular $(7.2 \%)$ groups, the difference was not statistically significant $(p=0.132)$. Similarly, distant metastasis was over 3-fold as common in the papillary $(11.8 \%)$ as in the tubular $(3.7 \%)$ group, the difference did not reach a statistically significant level $(\mathrm{p}=$ 0.067). Importantly, the overall 5-year survival rate was significantly worse in the papillary $(80.5 \%)$ group, compared to the tubular (96.8\%; Figure 3) $(p<$ 0.05).

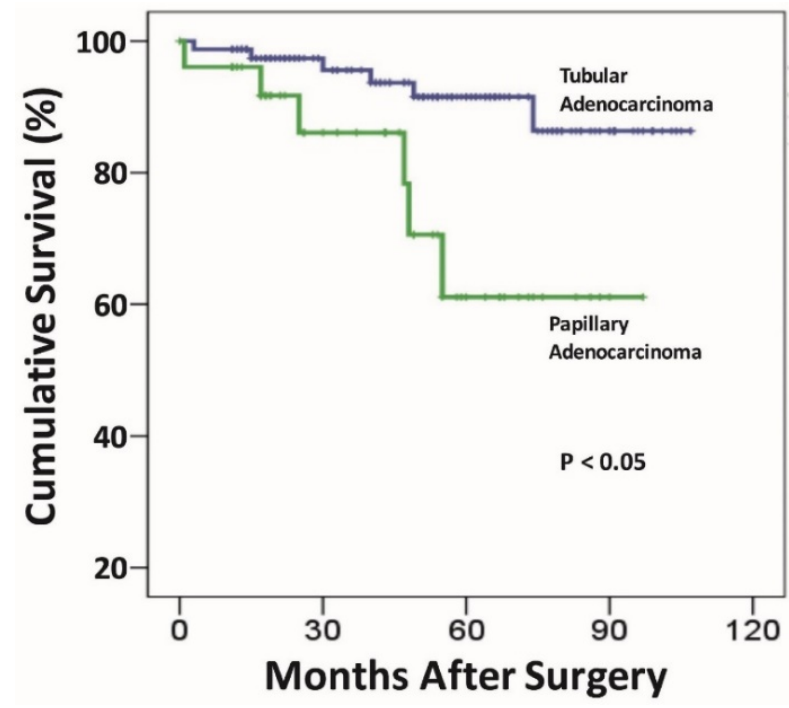

Figure 3. Comparison of the post-resection prognosis exhibits a statistically significant worse survival for the papillary early gastric adenocarcinoma group, compared to that of the tubular early gastric adenocarcinoma group.

Table 1. Comparison of clinical, endoscopic, and pathologic features between papillary and tubular early adenocarcinoma.

\begin{tabular}{|c|c|c|c|}
\hline Feature & Papillary $(n=59)(\%)$ & $\begin{array}{l}\text { Tubular }(\mathrm{n}=181) \\
(\%)\end{array}$ & $\mathrm{P}$ \\
\hline Age (years) & $65.9 \pm 6.9$ & $62.4 \pm 10.1$ & \\
\hline$\leq 60$ & $13(22.0)$ & $75(41.4)$ & 0.007 \\
\hline$\geq 61$ & $46(78.0)$ & $106(58.6)$ & \\
\hline \multicolumn{4}{|l|}{ Gender } \\
\hline Male & $43(72.9)$ & $130(71.8)$ & 0.875 \\
\hline Female & $16(27.1)$ & $51(28.2)$ & \\
\hline \multicolumn{4}{|l|}{ Tumor location } \\
\hline Proximal (Cardia) & $33(55.9)$ & $52(28.7)$ & $<0.001$ \\
\hline Distal (non-cardia) & $26(44.1)$ & $129(71.3)$ & \\
\hline \multicolumn{4}{|l|}{ Tumor size (cm) } \\
\hline$<2$ & $35(59.3)$ & $117(64.6)$ & 0.230 \\
\hline$\geq 2$ & $24(40.7)$ & $64(35.4)$ & \\
\hline Endoscopic gross type & & & $<0.001$ \\
\hline O-I & $22(37.3)$ & $13(7.2)$ & \\
\hline O-IIa & $17(28.8)$ & $58(32.0)$ & \\
\hline $\mathrm{O}-\mathrm{IIb}$ & $4(6.8)$ & $4(2.2)$ & \\
\hline O-IIc & $4(6.8)$ & $38(21.0)$ & \\
\hline O-III & $12(20.3)$ & $68(37.6)$ & \\
\hline Tumor invasion depth & & & 0.048 \\
\hline M2 & $9(15.3)$ & $49(27.1)$ & \\
\hline M3 & $12(20.3)$ & $53(29.3)$ & \\
\hline SM1 & $21(35.6)$ & $46(25.4)$ & \\
\hline SM2 & $17(28.8)$ & $33(18.2)$ & \\
\hline \multicolumn{4}{|l|}{ Micropapillary pattern } \\
\hline Present & $4(6.8)$ & $0(0)$ & 0.003 \\
\hline Absent & $55(93.2)$ & $181(100)$ & \\
\hline \multicolumn{4}{|c|}{ Lymphovascular invasion } \\
\hline Present & $10(16.9)$ & $15(8.3)$ & 0.059 \\
\hline Absent & $49(83.1)$ & $166(91.7)$ & \\
\hline \multicolumn{4}{|l|}{ Perineural invasion } \\
\hline Present & 0 & $2(1.1)$ & $>0.999$ \\
\hline Absent & $59(100)$ & $179(98.9)$ & \\
\hline Distant Metastasis & $(n=51)$ & $(n=162)$ & \\
\hline Present & $6(11.8)$ & $6(3.7)$ & 0.067 \\
\hline Absent & $45(88.2)$ & $156(96.3)$ & \\
\hline \multicolumn{4}{|l|}{ Lymph Node Metastasis } \\
\hline Present & $8(13.6)$ & $13(7.2)$ & 0.132 \\
\hline Absent & $51(86.4)$ & $168(92.8)$ & \\
\hline 5-year survival rate & $80.50 \%$ & $96.8 \%$ & 0.016 \\
\hline
\end{tabular}




\section{Differences in Risk Factors of Lymph Node Metastasis}

By univariate analysis, advanced age, male gender, tumor proximal gastric location, and tumor size over $2 \mathrm{~cm}$ were not statistically significant risk factors for nodal metastasis in either the papillary or tubular EGC group (Table 2). The risk of nodal metastasis for the endoscopic ulcerative pattern was significant only in the tubular $(p<0.05)$, but not in the papillary group. Similarly, submucosal invasion was a significant risk factor of nodal metastasis also only in the tubular $(p<0.05)$, but not in the papillary group. In contrast, the risk of nodal metastasis in cases with the micropapillary pattern approached, but did not reach, the statistically significant level in the papillary $(p=0.054)$, but not in the tubular group. As previously indicated [9], lymphovascular, but not perineural, invasion exhibited a significant risk of over 35-fold in papillary and over 36-fold in tubular groups for nodal metastasis $(\mathrm{p}<0.001)$.

\section{Independent Risk Factors of Lymph Node Metastasis}

Table 3 showed the results of multivariate analysis of 3 key risk factors related to the risk of lymph node metastasis in papillary and tubular EGCs. Neither the advanced age nor the micropapillary growth pattern was found to be a statistically significant risk factor for nodal metastasis. In contrast, lymphovascular invasion showed over 31-fold risk of nodal metastasis in papillary EGCs, higher than that (26.5-fold) in tubular EGCs.

Table 2. Risk factors of lymph node metastasis between patients with early papillary or tubular adenocarcinoma Risk factors of lymph node metastasis between patients with early papillary or tubular adenocarcinoma.

\begin{tabular}{|c|c|c|c|c|}
\hline \multirow[t]{2}{*}{ Feature } & \multicolumn{2}{|c|}{ Papillary $(n=59)$} & \multicolumn{2}{|c|}{ Tubular $(\mathrm{n}=181)$} \\
\hline & OR $(95 \% \mathrm{CI})^{*}$ & $P$ & OR (95\% CI) & $P$ \\
\hline \multicolumn{5}{|l|}{ Age (year) } \\
\hline$\leq 60$ & $2.46(0.50-12.06)$ & 0.267 & $1.72(0.55-5.33)$ & 0.350 \\
\hline$\geq 61$ & Ref & & Ref & \\
\hline \multicolumn{5}{|l|}{ Gender } \\
\hline Male & Ref & & Ref & \\
\hline Female & $0.88(0.16-4.89)$ & 0.885 & $2.34(0.75-7.35)$ & 0.144 \\
\hline \multicolumn{5}{|l|}{ Tumor location } \\
\hline Proximal (Cardia) & Ref & & & \\
\hline Distal (non-cardia) & 7.18E8 (0 - ) & 0.998 & 1.81E8 (0 - ) & 0.997 \\
\hline \multicolumn{5}{|l|}{ Tumor size $(\mathrm{cm})$} \\
\hline$<2$ & Ref & & Ref & \\
\hline$\geq 2$ & $1.55(0.35-6.92)$ & 0.566 & $2.27(0.73-7.08)$ & 0.157 \\
\hline \multicolumn{5}{|l|}{ Endoscopic gross type } \\
\hline $\mathrm{O}-\mathrm{I} / \mathrm{IIa} / \mathrm{IIb} / \mathrm{IIc}$ & Ref & & Ref & \\
\hline O-III (ulcerated) & $0.52(0.06-4.68)$ & 0.559 & $4.16(1.23-14.08)$ & 0.022 \\
\hline \multicolumn{5}{|l|}{ Tumor invasion depth } \\
\hline M2 and M3 & Ref & & Ref & \\
\hline SM1 and SM2 & 4.31E8 (0-) & 0.998 & $18.09(2.30-142.39)$ & 0.006 \\
\hline \multicolumn{5}{|l|}{ Micropapillary pattern } \\
\hline Present & $8.17(0.97-69.10)$ & 0.054 & $2.21(0.68-7.17)$ & 0.185 \\
\hline Absent & Ref & & Ref & \\
\hline \multicolumn{5}{|l|}{ Lymphovascular invasion } \\
\hline Present & $35.25(5.28-235.25)$ & $<0.001$ & $36.80(9.55-141.88)$ & $<0.001$ \\
\hline Absent & Ref & & Ref & \\
\hline
\end{tabular}

* OR: Odds Ratio; CI, confidence interval; Ref: reference.

Table 3. Intendant risk factors of lymph node metastasis of early papillary versus tubular adenocarcinoma.

\begin{tabular}{|c|c|c|c|c|}
\hline \multirow[t]{2}{*}{ Feature } & \multicolumn{2}{|c|}{ Papillary } & \multicolumn{2}{|c|}{ Tubular } \\
\hline & OR $(95 \% \mathrm{CI})^{*}$ & $P$ & OR $(95 \% \mathrm{CI})$ & $P$ \\
\hline \multicolumn{5}{|l|}{ Tumor invasion depth } \\
\hline $\mathrm{M} 2$ and $\mathrm{M} 3$ & - & & Ref & \\
\hline SM1 and SM2 & - & & $6.43(0.72-57.37)$ & 0.096 \\
\hline \multicolumn{5}{|l|}{ Endoscopic gross type } \\
\hline O-I/IIa/IIb/IIc & - & & Ref & \\
\hline O-III & - & & $3.93(0.83-18.70)$ & 0.086 \\
\hline \multicolumn{5}{|l|}{ Micropapillary pattern } \\
\hline Present & $4.79(0.26-88.68)$ & 0.293 & - & \\
\hline Absent & Ref & & - & \\
\hline \multicolumn{5}{|c|}{ Lymphovascular invasion } \\
\hline Present & $31.11(4.49-215.38)$ & $<0.001$ & $26.45(5.58-125.43)$ & $<0.001$ \\
\hline Absent & Ref & & $\operatorname{Ref}$ & \\
\hline
\end{tabular}

* OR: Odds Ratio; CI, confidence interval; Ref: reference. 


\section{Discussion}

In this comparison study, our data illustrate significantly worse prognosis in papillary EGC with a significantly lower 5-year survival rate of $80.5 \%$, compared to $96.8 \%$ in tubular EGC. This surprising finding may be related to the more frequent presence of high risk factors for worse prognosis in papillary EGC. In our cohort, papillary EGCs, compared to tubular EGCs, show significantly deeper invasion into the submucosal layer, more frequent association with a micropapillary component, and probably more frequent lymphovascular invasion, although the $p$ value for lymphovascular invasion was 0.059 . These results are parallel to those of the previous similar studies on papillary EGC from Japan $[18,19]$ and Korea [13], in which submucosal invasion was reported to be over 20\% [13], and lymphovascular invasion was found in over $28 \%$ in Korean patients [13] and over $70 \%$ in Japanese patients [18]. Importantly, the nodal metastasis rate is alarmingly high and discovered in $13.6 \%$ of the current cohort and over $18 \%$ in a Korean study and $18.2 \%-29.2 \%$ in two Japanese studies [18,19], which is significantly higher than that of non-papillary EGCs [13,19], including tubular EGCs as shown in this report. Those results raise a serious legitimate concern on indiscriminate use of endoscopic resection, such as ESD $[7,8]$, for papillary EGC because nodal metastasis is a contraindication for endoscopic resection of EGC.

Although both papillary and tubular EGC tumors are believed to be similar in prognosis and risk of nodal metastasis [6], the recent investigations from others and ours show the results otherwise $[9,13,18]$. We demonstrated several significant differences in the present comparison study between these two groups of EGC in prognosis-associated risk factors, such as significantly older patient age, more frequent proximal gastric location, and more common association with a micropapillary component, in addition to deeper invasion and higher frequency of lymphovascular, nodal, and distant metastases, as aforementioned. In EGC, lymphovascular invasion has been confirmed repeatedly as the most important risk factor for nodal metastasis $[9,20,21]$. According to Sekiguchi et al, the independent risk factors for lymphovascular invasion include large tumor size, submucosal invasion, undifferentiated adenocarcinoma, and papillary EGC that has over 8-fold risk, even for intramucosal EGC $(60 \%, 3 / 5)$ [12]. Distant metastasis is also more common in papillary than in tubular EGCs, as reported previously for papillary gastric cancer [10], although the difference in the current study did not reach a statistically significant level because of the small sample size.
Given more aggressive pathobiologic behavior and higher risk of nodal and distant metastases than tubular EGCs, papillary EGCs need to be treated differently and individually $[13,18]$.

Despite scarce published studies on papillary EGC, the available evidence, primarily from Japan and Korea, exhibits significantly different molecular pathogenesis mechanisms between papillary and tubular EGC tumors. For instance, compared to tubular EGCs with a high expression of intestine-type molecular markers such as MUC2 and CDX2, papillary EGCs show a distinctly higher expression of gastric mucin such as MUC5AC and MUC6 [18,22]. The gastric mucin phenotype in papillary EGCs has been reported to be significantly correlated to lymphatic invasion, nodal metastasis, and abnormal E-cadherin gene expression [18]. Most recently, Sugimoto et al discovered a significantly higher frequency of microsatellite instability in half $(9 / 18)$ papillary EGC tumors, compared to tubular EGCs [22]. Since both KRAS and BRAF gene mutations are rare in EGC, the microsatellite instability is suspected to be a major precursor lesion in EGC [22]. Apparently, further studies in larger samples from different ethnic populations are required to validate the findings that are important for patient management because of different prognosis and responses to 5-FU-based chemotherapy in gastric carcinoma with high microsatellite instability $[23,24]$.

The limitations of this study are several. 1).The retrospective study design inherits unavoidable selection bias, although consecutive cases were used in this study to minimize that shortcoming. 2). We followed the WHO guideline to select EGC tumors with a major papillary growth pattern in over $50 \%$ of the tumor volume for the comparison study. We excluded some cases with a minor papillary pattern in a mixed tumor, which may have different profiles affecting prognosis and risk of metastasis. 3) The sample size in the current study is small with unsatisfactory statistical results in $p$ values that approach the threshold for several risk factors. To validate the study results, we are currently conducting large-scale multicenter studies with the goal to illustrate accurately clinicopathology and prognosis of papillary EGCs in comparison with other types of EGCs for a better individualized EGC patient treatment strategy.

\section{Conclusions}

Papillary EGCs are uncommon but has higher propensity in elderly patients, proximal gastric location, elevated growth pattern, deeper invasion, higher lymphovascular invasion frequency, more nodal and distant metastases, and worse overall 
5-year survival rates, compared with tubular EGCs $[9,13,18,20]$. Because papillary EGCs can be easily identified by routine pathology evaluation, its high risk characteristics for worse prognosis should be used to guide clinical practice. Since most published studies on papillary EGCs, including our own, are based on single-center experience with small samples, it is urgent to perform systematic investigation of papillary EGCs in multicenter studies for an optimal clinical management of EGC patients.

\section{Competing Interests}

All authors have declared no competing interest.

\section{References}

1. Lauwers GY, Carneiro F, Graham DY, et al. Gastric carcinoma. In: Bosman FT, Carneiro F, Hruban RH, Theise ND, editors. WHO Classification of Tumours of the Digestive System. Lyon: IARC Press; 2010: 48-58.

2. Lauren $P$. The two histological main types of gastric carcinoma: diffuse and so-called intestinal-type carcinoma. An attempt at a histo-clinical classification. Acta Pathol Microbiol Scand 1965; 64:31-49.

3. Japanese classification of gastric carcinoma: 3rd English edition. Gastric Cancer 2011; 14:101-12

4. Torre LA, Bray F, Siegel RL, et al. Global cancer statistics, 2012. CA Cancer J Clin. 2015 Mar;65(2):87-108. doi: 10.3322/caac.21262. Epub 2015 Feb 4.

5. Chen W, Zheng R, Zhang S, et al. Report of incidence and mortality in China cancer registries, 2009. Chin J Cancer Res 2013; 25:10-21.

6. Gotoda T, Yanagisawa A, Sasako M, et al. Incidence of lymph node metastasis from early gastric cancer: estimation with a large number of cases at two large centers. Gastric Cancer 2000; 3:219-225.

7. Japanese gastric cancer treatment guidelines 2010 (ver. 3). Gastric Cancer 2011; 14:113-23.

8. Chinese digestive endoscopy society and anti-cancer endoscopy society. 2014 consensus opinions on early gastric cancer screening and therapy. Chin J Dig Endosc 2014; 31:361-77.

9. Huang Q, Fang C. Shi J, et al. Differences in Clinicopathology of Early Gastric Carcinoma between Proximal and Distal Location in 438 Chinese Patients. Scientific Report. 2015 Aug 27; 5:13439. doi: 10.1038/srep13439.

10. Yasuda K, Adachi Y, Shiraishi N, Maeo S, Kitano S. Papillary adenocarcinoma of the stomach. Gastric Cancer. 2000 Aug 4;3(1):33-38.

11. Kang HJ, Kim DH, Jeon TY, et al. Lymph node metastasis from intestinal-type early gastric cancer: experience in a single institution and reassessment of the extended criteria for endoscopic submucosal dissection. Gastrointest Endosc. 2010 Sep;72(3):508-15. doi: 10.1016/j.gie.2010.03.1077. Epub 2010 Jun 15.

12. Sekiguchi M, Sekine S, Oda I, et al. Risk factors for lymphatic and venous involvement in endoscopically resected gastric can- cer. J Gastroenterol. 2013; 48:706-12.

13. Lee HJ, Kim GH, Park do Y, et al. Is endoscopic submucosal dissection safe for papillary adenocarcinoma of the stomach? World J Gastroenterol. 2015 Apr 7;21(13):3944-52. doi: 10.3748/wjg. v21.i13.3944.

14. Everett SM, Axon AT. Early gastric cancer: disease or pseudo-disease? Lancet 1998;351: 1350-1352.

15. The Paris endoscopic classification of superficial neoplastic lesions: esophagus, stomach, and colon: November 30 to December 1, 2002. Gastrointest Endosc 2003;58: S3-43.

16. Huang Q, Shi J, Sun Q, et al. Clinicopathologic Characterization of Small $(\leq 2$ $\mathrm{cm})$ Proximal and Distal Gastric Carcinomas in a Chinese Population. Pathology. 2015; 47(6):526-32.

17. Lagergren J, Bergstrom R, Lindgren A Nyrén O. Symptomatic gastroesophageal reflux as a risk factor for esophageal adenocarcinoma. $N$ Engl J Med 1999; 340:825-31.

18. Koseki K, Takizawa T, Koike M, et al. Distinction of differentiated type early gastric carcinoma with gastric type mucin expression. Cancer. 2000; 89:724-32.

19. Sekiguchi M, Kushima R, Oda I, et al. Clinical significance of a papillary adenocarcinoma component in early gastric cancer: a single-center retrospective analysis of 628 surgically resected early gastric cancers. J Gastroenterol. 2015;50(4):424-34.

20. Gertler R, Stein HJ, Schuster T, Rondak IC, Höfler H, Feith M. Prevalence and topography of lymph node metastases in early esophageal and gastric cancer. Ann Surg. 2014; 259:96-101.

21. Fang C, Shi J, Sun Q, et al. Risk Factors of Lymph Node Metastasis in Early Gastric Carcinomas Diagnosed with WHO Criteria in 379 Chinese Patients. J Dig Dis. 2016 Jul 19. doi: 10.1111/1751-2980.12385. [Epub ahead of print]

22. Sugimoto R, Sugai T, Habano W, et al. Clinicopathological and molecular alterations in early gastric cancers with the microsatellite instability-high phenotype. Int J Cancer. 2016; 138:1689-97.
23. Leung SY, Yuen ST, Chung LP, Chu KM, Chan AS, Ho JC. hMLH1 promoter methylation and lack of hMLH1 expression in sporadic gastric carcinomas with high-frequency microsatellite instability. Cancer Res. 1999; 59:159-64.

24. An JY, Kim H, Cheong JH, Hyung WJ, Kim H, Noh SH. Microsatellite instability in sporadic gastric cancer: its prognostic role and guidance for 5-FU based chemotherapy after R0 resection. Int J Cancer. 2012;131:505-11. 